\title{
Exercise in Myositis
}

\section{Helene Alexanderson, PhD, RPT}

\author{
Address \\ Department of NVS, Huddinge, Karolinska Institutet and Department of Medicine, \\ Karolinska Institutet, Solna and Function Area Occupational Therapy and Physical \\ Therapy, Karolinska University Hospital, SE-171 76, Stockholm, Sweden \\ Email: Helene.alexanderson@sll.se \\ Published online: 23 November 2018 \\ (C) The Author(s) 2018
}

This article is part of the Topical Collection on Other CTD: Inflammatory Myopathies and Sjögren's

Keywords Dermatomyositis - Exercise $\cdot$ Inclusion body myositis $\cdot$ Polymyositis

\begin{abstract}
Purpose of the review A growing body of evidence supports exercise as a very important part of the treatment for adult patients with idiopathic inflammatory myopathies (IIM). This review mainly focuses on exercise studies published during the last 2 years in adult myositis.

Recent findings During the last couple of years, new publications present further evidence for intensive endurance exercise as an anti-inflammatory treatment inducing muscle growth and improving mitochondrial function compared a non-exercising control group. Further, blood-flow restricted resistance training was effective to maintain muscle strength compared to a non-exercising control group which lost a mean of $9.2 \%$ in quadriceps strength over 3 months in inclusion body myositis. Another study evaluates the effects of intra-muscular injections of an isoform of follistatin (FS344) by AAV1 in combination with exercise in a small group of patients with inclusion body myositis. An improvement in physical capacity was associated to higher exercise levels. Less is known about exercise effects in patients with recent onset, active polymyositis, and dermatomyositis.

Summary All studies report safety of exercise in all types and stages of myositis and exercise could now be considered as medicine. It is recommended to initiate exercise on a low intensity under supervision of a physical therapist with regular follow-up and progression of intensity over time.
\end{abstract}

\section{Introduction}

\section{The idiopathic inflammatory myopathies and effects of exercise}

The inflammatory myopathies are a heterogenous group of disorders divided into sub-groups adult polymyositis (PM), dermatomyositis (DM), and inclusion body myositis (IBM), as well as the juvenile dermatomyositis (JDM). Muscle impairments are the cardinal symptom in IIM, but involvement of extra-muscular organ systems is common, such as interstitial lung disease (ILD) and skin rash in DM. Patients with PM and DM present with reduced muscle strength, but more so with reduced muscle endurance [1]. Despite an often favorable effect of medical treatment, most patients develop sustained disability and reduced quality of life $[2,3]$. Patients have reduced aerobic capacity compared to healthy, matched controls [4,5]. Fatigue, pain, and cognitive impact are other important disease consequences that have not been much addressed in the literature [6]. 
Over the last two decades, many studies have been conducted to investigate safety and effects of exercise in adults with myositis, with the majority focusing on poly-

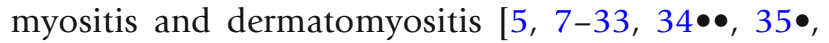
$36 \bullet \bullet$. The emerging evidence supports exercise as a safe and effective treatment to optimize health and reduce disability in adult patients with myositis. Excitingly, intensive exercise can even reduce disease activity and inflammatory markers as well as to improve muscle tissue health in adult polymyositis and dermatomyositis [4, $16,32,34 \bullet \bullet, 37]$. Exercise has become an important part of the treatment in both adult and juvenile IIM. This review will mainly focus on studies published during the last 2 years in adult patients with IIM.

\section{Effects of exercise in adult PM/DM}

\section{Disease activity, inflammation, and muscle health}

Two studies have reported on effects of exercise on disease activity and inflammation in adults with polymyositis and dermatomyositis. Two of eight patients with established polymyositis and dermatomyositis were responders with reduced disease activity [16] according to the established criteria ( $>20 \%$ improvement in $>3 / 6$ variables, with worsening $>20 \%$ in no more than two variables which cannot include the Manual Muscle Test, MMT) [38]. Micro array analysis of muscle biopsies pre- and post-training revealed downregulation of genes related to inflammation and fibrosis compared to baseline [19]. A randomized controlled trial evaluating an intensive aerobic and endurance-based exercise program supported the hypothesis that intensive exercise does not only improve objective and patientreported function and quality of life, but also can reduce disease activity in established PM and DM [5, 32]. The program consisted of 30 min of stationary biking on $70 \%$ of maximal oxygen uptake $\mathrm{VO}_{2 \max }$ together with endurance-based resistance training performed 3 days a week for 12 weeks. This type of exercise induced several positive changes in clinical disease activity both on a group level and, according to the responder criteria, and improved muscle metabolism that have been reviewed elsewhere $[39,40]$. New publications report on changes in inflammatory activity and in muscle health that could explain the improvements in physical capacity and quality of life by this intensive aerobic exercise program (Table 1 ).
Pre- and post-exercise muscle biopsies were available for a sub-group of patients from this study. The intensive exercise program induced a downregulation of protein degradation and in genes related to inflammation, immune response, and ER-stress. In addition, upregulation of genes related to muscle growth and signs of capillary growth. No similar response was identified in the non-

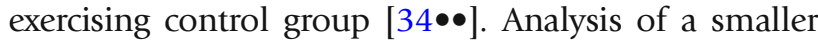
sub-set of patients from the RCT by Alemo Munters et al. (2013) [32] revealed changes in microRNAs targeting transcripts and proteins that are important for immune and muscle response $[41 \bullet \bullet$. These studies further support the hypothesis that exercise is a diseasemodifying treatment and an important part of the treatment in patients with PM and DM.

\section{Disability and quality of life}

The intensive aerobic exercise as described by Alemo 2013 et al. [32] significantly improved aerobic capacity and muscle function compared to the non-exercising control group [4, 32]. As expected, the aerobic biking program and the muscle endurance training resulted in foremost improved aerobic capacity assessed as the $\left(\mathrm{VO}_{2 \max }\right)$ and the biking time to exhaustion on $70 \%$ of maximal effort [4]. The exercise group also improved significantly in patient-reported physical function and vitality (SF-36) compared to the control group and there was additional within-exercise group improvement in SF-36 General Health and Mental health while the control group was unchanged. $\mathrm{VO}_{2 \max }$ and patient-reported physical function correlated strongly both at baseline and after 12 weeks of exercise indicating that aerobic capacity is an important variable controlling patientreported health. Quadriceps muscle strength was also improved in the exercise group as assessed by the five voluntary repetition maximum (the weight the patient can lift a maximal of five times), compared to the control group and interestingly, this variable was the only to be consistently improved in the exercise group at the one-year follow-up, while all other variables returned to baseline values. Alemo et al. also investigated the effects of exercise on activity limitations (ability to perform daily activities). Self-reported ability to walk, climb stairs, and run (myositis activities profile sub-scale moving around) was significantly improved in the exercise group compared to the control group with additional exercise group improvement in sub-scales social activities and leisure activities. In addition, patient-preference 


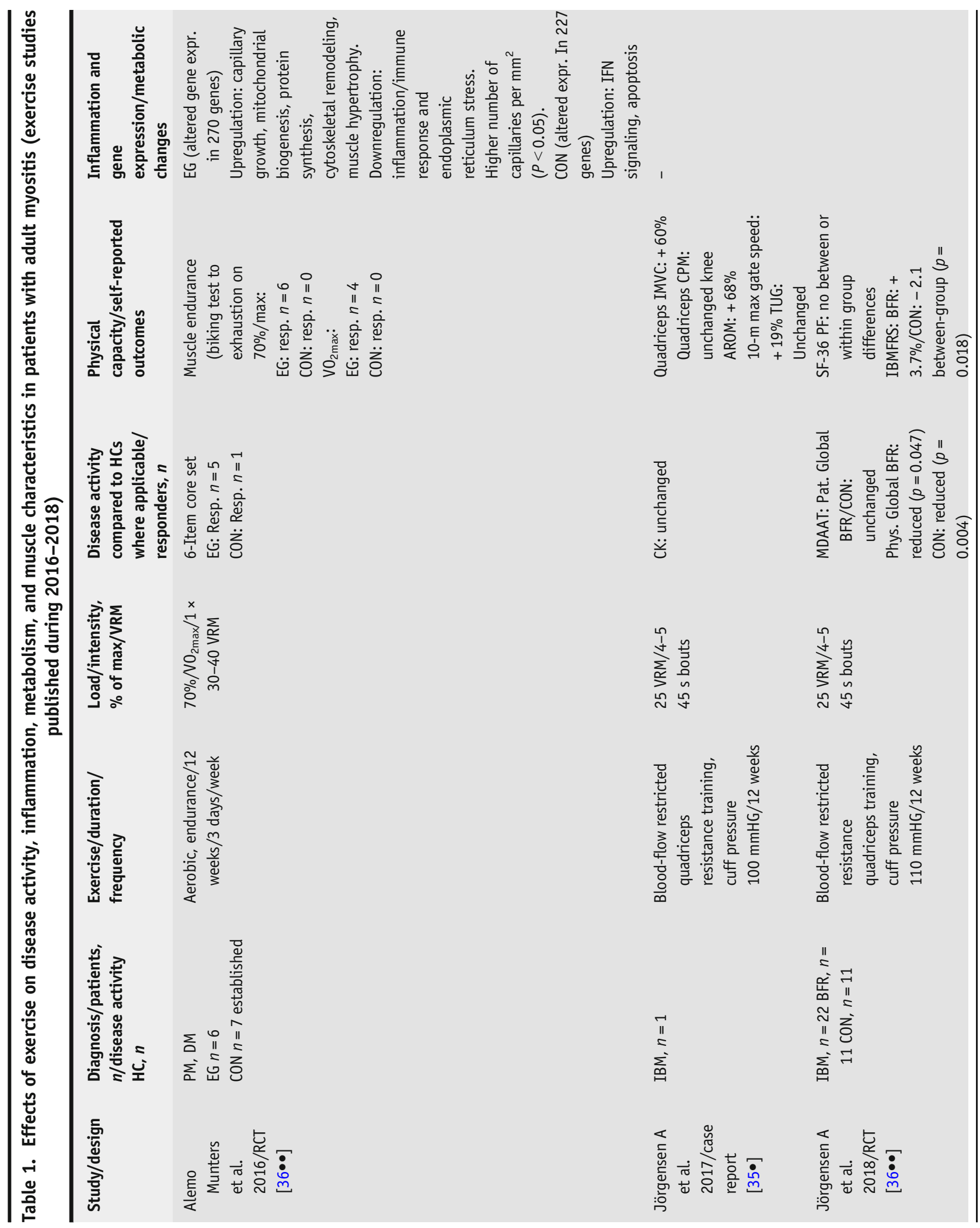




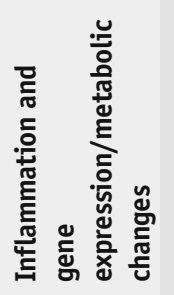

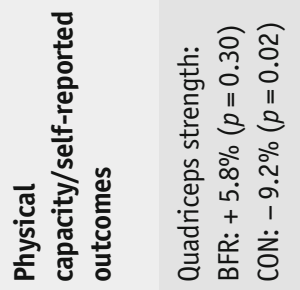

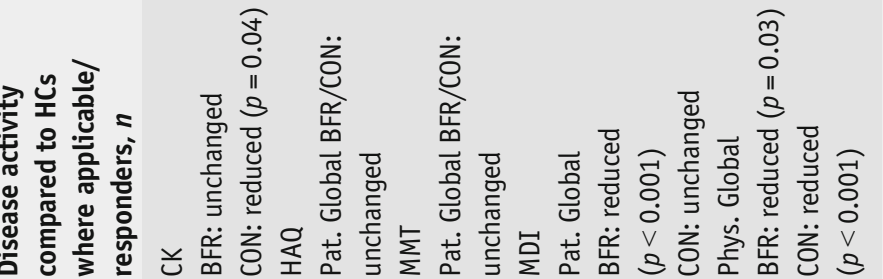

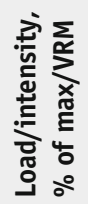

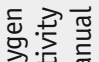

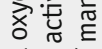

獄

离

है:

9 空

要这要

它全

污

은 등

客

음 ㅎّㅇ 두

造 둔욱

잉

응

옹으을

등

३콘든

齐

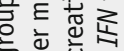

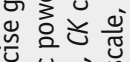

这艺

心 छ

บ

혼 $\frac{\pi}{8}$ 윰

응은돈

골음

证

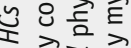

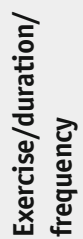

ज语 형

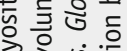

要

궁동

응

号氙

经的

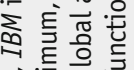

次完它

全呪

웅

톤원

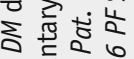

究客它

於艺苍

ह

范苍苍 
activities were also improved by exercise in the exercise group. According to available evidence in patients with established polymyositis and dermatomyositis, exercise recommendations for patients with myositis do not differ significantly from established recommendations of exercise to improve muscle function and aerobic capacity (Table 2).

Only a few studies have included patients with recent onset, active polymyositis, and dermatomyositis and no study with this focus has been published during the last 2 years. A home exercise program on low or moderate intensity (adapted to individual disease activity and disability) has been extensively investigated in patients with recent onset disease [12]. As the program can be individually adapted, it is well tolerated by all patients. The program was evaluated in combination with moderateintensity walks 5 days a week in a randomized controlled trial supporting the safety but was not able to show additional short-term positive effects on muscle function and aerobic capacity in combination with standard medical treatment compared to a control group receiving medical treatment alone [33]. However, the exercise group was at 1 and 2 years follow-up more physically active and had improved physical capacity compared to the control group. This implies that it is important to start exercise early to have a physically active lifestyle and improved physical capacity later in the disease course. The hypothesis is that a more intensive exercise program is needed to achieve improved physical capacity and quality of life in addition to the medical treatment. In background of new knowledge of the anti-inflammatory effects of exercise proven in patients with an established myositis, it is of outmost importance to focus research on safety and effects of exercise early in the disease course. A randomized controlled trial investigating clinical and molecular effects of high-intensity interval training is ongoing at the Karolinska University Hospital (clinicaltrial.gov ID NCT03324152).

\section{Exercise effects in sIBM}

Two case reports exploring feasibility of blood-restricted resistance training (BRF) in sporadic inclusion body myositis indicated positive effects on muscle strength $[22,35 \bullet$. A randomized controlled trial from group in Denmark was not able to show improvement in selfreported or objectively assessed function in the exercise

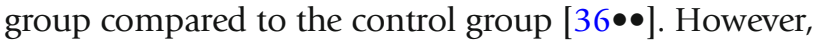
there was a striking significant time effect difference regarding knee extensor muscle strength in favor for the exercise group which was unchanged $(+5.8 \%)$ while the control group worsened $(-9.2 \%)$. This time effect difference was also true for the self-reported physical function assessed by the IBM functional rating scale indicating that exercise slows down disease progression and maintains function and physical independence. Individuals with IBM were randomized either to the BRF group exercising twice a week for 12 weeks or to a nonexercising control group. The BRF group performed supervised unilateral knee extensions in a leg press in three sets of $25 \mathrm{VRM}$, allowing 25 repetitions until exhaustion with a fourth set introduced at week 9 . Four weeks into the exercise program, knee flexion exercises were introduced according to the same protocol. The program also contained calf raise and dorsiflexion of the ankles. Blood flow was restricted by inflicting a $100 \mathrm{~mm}$ wide inflatable pneumatic cuff to $110 \mathrm{mmHG}$, connected to a computerized tourniquet system. The authors discussed that the exercise period of 12 weeks might have been too

Table 2. Recommendations for health-enhancing physical activity and exercise for healthy individuals [48]

\begin{tabular}{|c|c|c|c|c|}
\hline & $\begin{array}{l}\text { Duration of exercise } \\
\text { bouts, } \min \end{array}$ & $\begin{array}{l}\text { Intensity, } \\
\text { percentage of VRM }\end{array}$ & $\begin{array}{l}\text { Intensity, percentage of age predicted } \\
\text { maximal heart rate }\end{array}$ & $\begin{array}{l}\text { Frequency, } \\
\text { times/week }\end{array}$ \\
\hline $\begin{array}{l}\text { Increase muscle } \\
\text { strength }\end{array}$ & - & $60-80$ & - & $2-3$ \\
\hline $\begin{array}{l}\text { Increase muscle } \\
\text { endurance }\end{array}$ & - & $30-40$ & - & $2-3$ \\
\hline $\begin{array}{l}\text { Increase aerobic } \\
\text { capacity }\end{array}$ & $30-60$ & - & $60-85$ & 3 \\
\hline $\begin{array}{l}\text { Improve/maintain } \\
\text { health }\end{array}$ & 30 & $50-70$ & - & $4-7$ \\
\hline
\end{tabular}


short to be able to achieve improvements and that the study was slightly under-powered. In addition, there were within-patient differences in exercise response. Patients with less disease duration had a better response indicating the importance of introducing exercise as a treatment early in the disease course with a possibility to postpone damage and disability. Authors discuss further that the reduced disease activity seen in both groups was likely related to interrater variability and not a result of the intervention. The 16-week home exercise program that was evaluated in an open-design study including seven patients with IBM did achieve significant and clinically relevant improvements in knee extensor strength and functional tests such as $30-\mathrm{m}$ walk and sit-to-stand test [30]. Clinical experience from using this program at the Karolinska University Hospital supports these positive results to some extent. Similarly, to the RCT by Jörgensen et al., clinical experience indicates that some patients with IBM respond very well to treatment, improving both in strength and functional capacity, while the large majority experience small improvements in one functional test. However, as reported by Jörgensen et al. [36••] and based on clinical experience, few patients might not tolerate exercise well with increased muscle weakness or increased pain as a result of exercise. Today, we are not able to predict which patients with IBM will tolerate and respond to exercise or not, and a relevant question is if immunosuppressive treatment increases the chances to respond to treatment? Furthermore, there is an urgent need for larger RCTs evaluating different exercise programs to understand what type of exercise is the most efficacious and what factors might predict response to exercise. Recently, a large RCT investigated the effects of the myostatin inhibitor Bimgrabumab in IBM [42]. Primary outcome was the 6-min walking test, which was not altered compared to the placebo-controlled group and the study was stopped after 1-year follow-up. Levels of exercise or physical activity among participants in both groups were not registered. Mendell et al. [43•] treated five patients with IBM with intra-muscular injections of an isoform of follistatin (FS344) by AAV1 in combination with exercise [43•]. All patients were encouraged to exercise as much as possible but to do a minimum of 15-min stationary biking and knee extensor resistance training 3 days a week for the study period of 1 year. At 1-year follow-up, the treatment group was significantly and clinically relevant improved in the primary outcome 6MWT compared to a non-treated control group matched for age, disease duration, and baseline 6MWT.
Further, a larger improvement in 6MWT was associated to regular exercise habits. Based on this, it would be interesting to test Bimgrabumab in combination with a specific exercise program. As of today, we do not know what exercise regimen is most effective in IBM; however, evidence supports safety of individually adapted exercise and that regular exercise can maintain function while

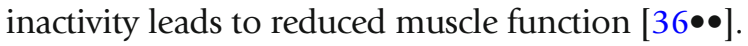

\section{Specific hand exercise programs in all adult patients with myositis}

It is well-known that a progressing, severe muscle weakness of finger and wrist flexors is a cardinal symptom in inclusion body myositis. An ongoing hand exercise study at the Karolinska University Hospital in Stockholm will hopefully soon improve our understanding on how individualized specific hand exercise can improve grip strength and hand function in patients with inclusion body myositis. Grip strength is also reduced in patients with adult polymyositis and dermatomyositis compared to reference values (2) and a specific hand exercise program could improve both grip strength and dexterity in these patients (regard hand exercise). Individualized hand exercise is therefore also an important part of the treatment in myositis and should be initiated ideally by an occupational therapist with knowledge of myositis.

\section{Discussion}

During the last 2 years, a few new publications have emerged improving our understanding of the effects of exercise on muscle health and inflammation. Exercise can improve mitochondrial function, angiogenesis as well as improve muscle growth and reduce inflammation in established polymyositis and dermatomyositis. Further, a randomized control trial was recently published that strongly supports the importance to exercise with the purpose to maintain or in some cases also improve muscle function in patients with inclusion body myositis.

Exercise reduces systemic inflammation in healthy individuals [44] and studies in other inflammatory conditions such as rheumatoid arthritis and systemic lupus erythematosus also indicate less inflammation by exercise [37]. Thus, it is not surprising that intensive exercise has shown the same positive effects in patients with polymyositis and dermatomyositis. The notion that exercise could be harmful in myositis was based on studies 
in healthy athletes performing strenuous types of exercise such as a Marathon race [39]. An acute response with increased serum creatine phosphokinase levels (CK) is normal and levels go back to baseline levels within $24 \mathrm{~h}$. There will also be an intra-muscular inflammatory response, with increased IL6-levels, which is part of the regeneration leading to improved muscle function [37]. All exercise studies in myositis report unchanged CK levels over weeks as well as unchanged or somewhat lower inflammation after exercise assessed by MRI or analysis of muscle biopsies [11, 12, 16, 32] in both established and early, active disease. Thus, individually adapted exercise should be introduced within weeks from starting medical treatment with frequent followup, at least every third month, during the first year. Exercise also needs to be progressed regularly according to changes in disease activity and physical capacity.

A recent study reports mitochondrial abnormalities in patients with recent onset untreated dermatomyositis with increased reactive oxygen species (ROS) production and interferon type I signature in muscle tissue correlating to reduced exercise capacity [45]. These data support introduction of exercise early in the disease course to counteract muscle pathology in muscle and hopefully restoring muscle function and muscle health sooner and to a larger extent than today. In addition, exercise could reduce corticosteroid-induced muscle atrophy and osteoporosis. However, more studies are needed.

The few studies of exercise effects in inclusion body myositis as well as clinical experience indicate that these patients might need to exercise more frequently to be able to achieve clinically meaningful improvements in the most affected muscle groups (Johnson et al. 2007), than patients with polymyositis or dermatomyositis. The study by Jörgensen et al. 2017 [36••] is very important as the first study to describe the natural course of deterioration of muscle weakness compared to twice a week exercise in inclusion body myositis, strongly supporting the role of exercise as a treatment to maintain and possibly also improve muscle strength in these patients. Only one case report has included analysis of muscle biopsies before and after blood-flow restricted resistance training in one patient with inclusion body myositis revealing slightly increased muscle fiber area and no signs of increased inflammation or damage [31]. Further research is needed to understand muscle pathology in inclusion body myositis and how exercise could improve muscle health in these patients.
The concept to use improvement criteria to study individual exercise response is interesting. Myositis is a heterogeneous disease where many factors can influence exercise response. Tolerance and response to medical treatment and the disease duration until diagnosis and subsequent degree of damage are two factors that may influence exercise response. Further, different antibody profiles and different disease mechanisms probably also play a role. The first improvement criteria for disease activity were proven useful to identify individuals responding to exercise with reduced disease activity [16, 32]. In addition, the ACR 20,50, and 70\% responder criteria [46] could identify patients responding in muscle function, aerobic capacity, and activity limitations by exercise $[16,32]$. In addition, new revised response criteria for disease activity were recently published [47]. Using these criteria in addition to group analysis will enhance individualized treatment by understanding what patients respond to different types of exercise. Clinical experience suggests a variation in exercise response in patients with inclusion body myositis. Again, diagnosis duration and degree of damage are two important factors, but there are probably different subtypes of inclusion body myositis with varying muscle involvement and progression time and knowledge of the roles of antibodies is very limited. Our own clinical experience indicates that most patients tolerate exercise well with limited improvements or mostly maintaining function. A few patients seem to respond very well with larger improvements in function and tolerating a very high exercise frequency and intensity, while yet a smaller group of patients might not tolerate even mild exercise. There is an urgent need for more, larger studies looking into exercise in inclusion body myositis.

In summary, exercise is a very important part of the treatment in adult patients with myositis to optimize function and quality of life as well as disease activity. Patients with recent onset disease require regular followup and adaption of exercise to changes in physical capacity, fatigue, and pain and exercise should ideally be initiated and followed up by a physical therapist with knowledge of myositis. Specific hand exercise is also helpful to optimize function in patients with myositis. Myositis is a multi-organ system, heterogeneous disease and thus requires an expert team of medical and rehabilitation health professionals to optimize prognosis and quality of life. 


\title{
Compliance with Ethical Standards
}

\author{
Conflict of Interest \\ Dr. Alexanderson declares no conflicts of interest.
}

Human and Animal Rights and Informed Consent

This article does not contain any studies with human or animal subjects performed by any of the authors.

Open Access This article is distributed under the terms of the Creative Commons Attribution 4.0 International License (http://creativecommons.org/licenses/by/4.0/), which permits unrestricted use, distribution, and reproduction in any medium, provided you give appropriate credit to the original author(s) and the source, provide a link to the Creative Commons license, and indicate if changes were made.

\section{References and Recommended Reading}

Papers of particular interest, published recently, have been highlighted as:

- Of importance

$\bullet \quad$ Of major importance

1. Alexanderson $\mathrm{H}$, Regardt M, Ottosson C, Alemo Munters L, Dastmalchi M, Dani L, et al. Muscle strength and muscle endurance during the first year of treatment of polymyositis and dermatomyositis: a prospective study. J Rheumatol. 2018;45(4):538-46.

2. Regardt $M$, Welin Henriksson E, Alexanderson $\mathrm{H}$, Lundberg IE. Patients with polymyositis or dermatomyositis have reduced grip force and health-related quality of life in comparison with reference values: an observational study. Rheumatology (Oxford). 2011;50(3):578-85.

3. Poulsen $\mathrm{KB}$, Alexanderson $\mathrm{H}$, Dalgård C, Jacobsen $\mathrm{S}$, Weile L, Diederichsen LP. Quality of life correlates with muscle strength in patients with dermato- or polymyositis. Clin Rheumatol. 2017;36(10):2289-95.

4. Alemo Munters L, Dastmalchi M, Katz A, Esbjörnsson M, Loell I, Hanna B, et al. Improved exercise performance and increases aerobic capacity after endurance training of patients with stable polymyositis and dermatomyositis. Arthritis Res Ther. 2013;15(4):R83.

5. Wiesinger GF, Quittan M, Nuhr M, Volc-Platzer B, Ebenbichler G, Zehetgruber M, et al. Aerobic capacity in adult dermatomyositis/polymyositis patients and healthy controls. Arch Phys Med Rehabil. 2000;81(1):1-5.

6. Regardt M, Basharat P, Christopher-Stine L, Sarver C, Björn A, Lundberg IE, et al. Patients' experience of myositis and further validation of a myositis-specific patient reported outcome measure - establishing core domains and expanding patient input on clinical assessment in myositis. Report from OMERACT 12. J Rheumatol. 2015;42(12):2492-5.
7. Hicks JE, Miller F, Plotz P, Chen TH, Gerber L, et al. Isometric exercise increases strength and does not produce sustained creatinine phosphokinase increases in a patients with polymyositis. J Rheumatol. 1993;20(8):1399-401.

8. Escalante A, Miller L, Beardmore TD. Resistive exercise in the rehabilitation of polymyositis/dermatomyositis. J Rheumatol. 1993;20(8):1340-4.

9. Wiesinger GF, Quittan M, Aringer M, Seeber A, VolcPlatzer B, Smolen J, et al. Improvements of physical fitness and muscle strength in polymyositis/ dermatomyositis patients. Br J Rheumatol. 1998;37(2):196-200.

10. Wiesinger GF, Quittan, Graninger M, Seeber A, Ebenbichler G, Sturm B, et al. Benefit of 6 months longterm physical training in polymyositis/dermatomyositis patients. Br J Rheumatol. 1998;37(2):1338-42.

11. Alexanderson $\mathrm{H}$, Stenström CH, Lundberg I. Safety of a home exercise programme in patients with polymyositis and dermatomyositis: a pilot study. Rheumatology (Oxford). 1999;38(7):608-11.

12. Alexanderson $\mathrm{H}$, Stenström $\mathrm{CH}$, Jenner G, Lundberg I. The safety of a resistive home exercise program in patients with recent onset active polymyositis or dermatomyositis. Scand J Rheumatol. 2000;29(5):295301.

13. Heikkilä S, Viitanen JV, Kautiainen H, et al. Rehabilitation in myositis. Physiotherapy. 2001;87(xx):301-9.

14. Varju C, Pethö E, Kutas R. The effect of physical exercise following acute disease exacerbation in patients with dermato/polymyositis. Clin Rehabil. 2003; $17(\mathrm{xx}): 83-7$. 
15. Harris-Love MO. Safety and efficacy of submaximal eccentric strength training for a subject with polymyositis. Arthritis Rheum. 2005;53(xx):471-4.

16. Alexanderson H, Dastmalchi M, Esbjörnsson-Liljedahl $\mathrm{M}$, Opava $\mathrm{CH}$, Lundberg IE. Benefits of intensive resistance training in patients with chronic polymyositis or dermatomyositis. Arthritis Rheum. 2007;57(5):768-77.

17. Dastmalchi M, Alexanderson H, Loell I, Ståhlberg M, Borg K, Lundberg IE, et al. Effect of physical training on the proportion of slow-twitch type I muscle fibers, a novel nonimmune-mediated mechanism for muscle impairment in polymyositis or dermatomyositis. Arthritis Rheum. 2007;57(7):1303-10.

18. Chung YL, Alexanderson H, Pipitone N, Morrison C, Dastmalchi M, Ståhl-Hallengren C, et al. Creatine supplements in patients with idiopathic inflammatory myopathies who are clinically weak after conventional pharmacologic treatment: six-month, double-blind, randomized placebo-controlled trial. Arthritis Rheum. 2007;57(4):694-702.

19. Nader GA, Dastmalchi M, Alexanderson H, Grundtman C, Gernapudi R, Esbjörnsson M, et al. A longitudinal, integrated, clinical, histological and mRNA profiling study of resistance exercise in myositis. Mol Med. 2010;16(11-12):455-64.

20. Hejazi SM, Engkasan JP, Qomi MSM. Intensive exercise and a patient in acute phase of polymyositis. J Back Musculoskelet Rehabil. 2012;25(4):231-4.

21. Mattar MA, Gualano B, Perandini LA, Shinjo SK, Lima FR, Sá-Pinto AL, et al. Safety and possible effects of lowintensity resistance training associated with partial blood flow restriction in polymyositis and dermatomyositis. Arthritis Res Ther. 2014;16(5):473.

22. Mattar MA, Gualano B, Roschel H, Perandini LA, Dassouki T, Lima FR, et al. Exercise as an adjuvant treatment in persistent active polymyositis. J Clin Rheumatol. 2014;20(1):11-5.

23. Regardt M, Schult ML, Axelsson Y, Aldehag A, Alexanderson H, Lundberg IE, et al. Hand exercise intervention in patients with polymyositis and dermatomyositis: a pilot study. Musculoskelet Care. 2014;12(3):160-72.

24. Dalise S, Bertolucci F, Simonella C, Rossi B, Chisari C. Intensive aerobic training improves motor performances and oxidative metabolism efficiency in chronic polymyositis: a case report. Neuromuscul Disord. 2012;22(Suppl 3):221-5.

25. Karper WB, Hopewell R, Hodge M. Exercise program effects on one woman with dermatomyositis. Rehabil Nurs. 2001;26(4):129-31.

26. Spector SY, Lemmer JT, Koffman BM, Fleisher TA, Feuerstein IM, Hurley BF, et al. Safety and efficacy of strength training in patients with sporadic inclusion body myositis. Muscle Nerve. 1997;20(10):1242-8.

27. Arnardottis S, Alexanderson H, Lundberg IE, Borg K. Sporadic inclusion body myositis: pilot study on the effects of a home exercise program on muscle function, histopathology and inflammatory reaction. J Rehabil Med. 2003;35(1):31-5.

28. Bertolucci F, Neri R, Dalise S, Venturi M, Rossi B, Chisari C. Abnormal lactate levels in patients with polymyositis and dermatomyositis: the benefits of a specific rehabilitative program. Eur J Phys Rehabil Med. 2014;50(2):161-9.

29. Johnson LG, Edwards DJ, Walters S, Thickbroom GW, Mastaglia FL. The effectiveness of an individualized home-based functional exercise program for patients with sporadic inclusion body myositis. J Clin Neuromuscul Dis. 2007;8(4):187-94.

30. Johnson GL, Collier KE, Edwards DJ, Philippe DL, Eastwood PR, Walters SE, et al. Improvement in aerobic capacity after an exercise program in sporadic inclusion body myositis. J Clin Neuromuscul Dis. 2009;10(4):178-84.

31. Gualano B, Neves M Jr, Lima FR, Pinto AL, Laurentino $\mathrm{G}$, Borges $\mathrm{C}$, et al. Resistance training with vascular occlusion in inclusion body myositis: a case study. Med Sci Sports Exerc. 2010;42(2):250-4.

32. Alemo Munters L, Dastmalchi M, Andgren V, Emilson C, Bergegård J, Regardt M, et al. Improvement in health and possible reduction in disease activity using endurance exercise in patients with established polymyositis and dermatomyositis: a multicenter randomized controlled trial with a 1-year open extension follow-up. Arthritis Care Res. 2013;65(12):1959-68.

33. Alexanderson H, Munters LA, Dastmalchi M, Loell I, Heimbürger M. Opava, et al. Resistive home exercise in patients with recent-onset polymyositis and dermatomyositis - a randomized controlled single-blinded study with a 2-year followup. J Rheumatol. 2014;41(6):1124-32.

34.• Munters LA, Loell I, Ossipova E, Raouf J, Dastmalchi M, Lindroos E, et al. Endurance exercise improves molecular pathways of aerobic metabolism in patients with myositis. Arthritis Rheum. 2016;68(7):1738-50

This is the first study to report down-regulation of proinflammatory genes and up-regulation of anti-inflammatory genes and genes regulating muscle growth after intensive exercise compared to a non-exercising control group in established polymyositis and dermatomyositis.

35. Jørgensen AN, Aagaard P, Nielsen JL, Frandsen U, Diederichsen LP. Effects of blood-flow-restricted resistance training on muscle function in a 74-year-old male with sporadic inclusion body myositis: a case report. Clin Physiol Funct Imaging. 2016;36(6):504-9

This recent case report describes a man with sporadic inclusions body myositis who tolerates blood-flow restricted resistance training resulting in improved physical capacity.

$36 . \bullet$ Jørgensen AN, Aagaard P, Frandsen U, Boyle E, Diederichsen LP. Blood-flow restricted resistance training in patients with sporadic inclusion body myositis: a randomized controlled trial. Scand J Rheumatol. 2018;47(5):400-9

This is the first randomized controlled exercise study in sporadic inclusion body myositis, demonstrating that blood-flow restricted resistance training led to a maintained 
muscle strength compared to a non-exercising control group over a 3-month period.

37. Benatti FB, Pedersen BK. Exercise as an antiinflammatory therapy for rheumatic diseases - myokine regulation. Nat Rev Rheumatol. 2015;11(2):86-97.

38. Rider LG, Giannini EH, Brunner HI, Ruperto N, JamesNewton L, Reed AM, et al. International consensus on preliminary definitions of improvement in adult and juvenile myositis. Arthritis Rheum. 2004;50(4):2280-90.

39. Alexanderson H. Physical exercise as a treatment for adult and juvenile myositis. J Intern Med. 2016;280(1):75-96.

40. Alemo Munters L, Alexanderson H, Crofford LJ, Lundberg IE. New insights into the benefits of exercise for muscle health in patients with idiopathic inflammatory myositis. Curr Rheumatol Rep. 2014;16(7):429.

41.• Boehler JF, Hogarth MW, Barberio MD, Novak JS, Ghimbovschi S, Brown KJ, et al. Effect of endurance exercise on microRNAs in myositis skeletal muscle-a randomized controlled study. PLoS One. 2017;12(8):e0183292

This study provides further evidence of positive effects on muscle health compared by a non-exercising control group in established polymyositis and dermatomyositis.

42. Amato AA, Sivakumar K, Goyal N, David WS, Salajegheh M, Praestgaard J, et al. Treatment of sporadic inclusion body myositis with bimagrumab. Neurology. 2014;83(24):2239-46.

43.• Mendell JR, Sahenk Z, Al-Zaidy S, Rodino-Klapac LR, Lowes LP, Alfano LN, et al. Follistatin gene therapy for sporadic inclusion body myositis improves functional outcome. Molecular Ther. 2017;25(4):870-.

This study indicates that improvements by a myostatin blocker was associated to higher exercise levels in inclusion body myositis.
44. Cronin O, Keohane DM, Molloy MG, Shanahan F. The effects of exercise interventions on inflammatory biomarkers in healthy physically inactive subjects: a systematic review. QJM. 2017;110(10):629-37.

45. Meyer A, Laverny G, Allenbach Y, Grelet E, Ueberschlag $\mathrm{V}$, Echaniz-Laguna A, et al. IFN- $\beta$-induced reactive oxygen species and mitochondrial damage contribute to muscle impairment and inflammation maintenance in dermatomyositis. Acta Neuropathol. 2017;134(4):655-66.

46. Paulus HE, Egger MJ, Ward JR, Williams HJ. and the Cooperative Systematic Studies of Rheumatic Diseases Group. Analysis of improvement in individual rheumatoid arthritis patients treated with diseasemodifying antirheumatic drugs, based on the findings in patients treated with placebo. Arthritis Rheum. 1990;33(4):477-84.

47. Aggarwal R, Rider LG, Ruperto N, Bayat N, Erman B, Feldman BM, et al. 2016 American College of Rheumatology/European League Against Rheumatism criteria for minimal, moderate and major clinical response in adult dermatomyositis and polymyositis. An international myositis assessment and clinical studies group/paediatric rheumatology international trials organization collaborative initiative. Ann Rheum Dis. 2017;76(5):792-801.

48. Ewing Garber C, Blissmer B, Deschenes MR, Franklin BA, Lamonte MJ, Lee IM, et al. Quantity and quality of exercise for developing and maintaining cardiorespiratory, musculoskeletal, and neuromotor fitness in apparently healthy adults: guidance for prescribing exercise. Med Sci Sports Exerc. 2011;43(7):1334-59. 